\title{
Changes in solar irradiance in an 11-yr cycle and on a secular timescale: observations and reconstruction using neurocomputing
}

\author{
A.V. Mordvinov ${ }^{1}$ and N.G. Makarenko ${ }^{2}$ \\ ${ }^{1}$ Institute of Solar-Terrestrial Physics, P.O. Box 4026, Irkutsk, Russia email: avm@iszf.irk.ru \\ ${ }^{2}$ Institute of Mathematics, Almaty, Kazakhstan
}

Changes in Total Solar Irradiance (TSI) were studied both in time-frequency and in time-longitude aspects. The continuous wavelet analysis of TSI composite time series (Fröhlich \& Lean 1998; Willson \& Mordvinov 2003) revealed that the energy of thermomagnetic disturbances due to sunspots and faculae cascades into the magnetic network and facular macrostructure. The time-longitude analysis revealed large-scale patterns of radiative excesses in solar atmosphere. These patterns are organized into 2- and 4- sector structures exhibiting the effects of both activity complexes and magnetically active longitudes. Large-scale patterns with radiative excess display a facular macrostructure. These temperature patterns are causally related to long lived magnetic fields of the Sun. During activity cycles $21-23$ the patterns with radiative excess tend to be concentrated around the active longitudes which are centered at about $60^{\circ}$ and $230^{\circ}$ in the Carrington system (Benevolenskaya et al. 1999; Mordvinov \& Willson 2003).

Based on multicsale representation the Artificial Neural Networks (ANN) were developed to model and to reconstruct changes in TSI using the sunspot indices since 1610 . Structure of the TSI model was identified from the regression relation between the group sunspot numbers Rg (Hoyt \& Schatten 1998) and TSI. The cross-correlation function between the sunspot index and TSI is asymmetric and demonstrates non-linear causal relation between sunspot and radiant indices. The empirical ANN model approximates changes in TSI using its inputs: the sunspot index values with lags which describe shortterm prehistory of magnetic activity as well as the cyclic and long-term components of the wavelet decomposition of $\mathrm{Rg}$ time series. From numerical experiments the optimal tree-layers perceptron was found to model the TSI behavior. Using sunspot indices the regression ANN model was constructed and trained to approximate changes in TSI for 1610-2003.

Changes in solar luminosity on a secular timescale were derived using indirect data about magnetic activity of the Sun from natural archives of cosmogenic isotopes on the assumption of the stationary relation between the radiant and proxy indices. We used the cosmogenic isotope ${ }^{10} \mathrm{Be}$ record by Bard et al. 1997 to reconstruct changes in TSI. The regression ANN derives non-linear causal relation between solar luminosity and changes in the ${ }^{10} \mathrm{Be}$ abundance. The ANN technique provides an independent approach that qualitatively agree with previous TSI reconstructions (Lean et al. 1995; Solanki \& Fligge (1999); Usoskin et al. 2004). Figure 1 shows long-term changes in solar luminosity which are characterized by intermittent depressions due to the great minima of magnetic activity of the Sun. The deepest TSI minimum in the 15th century looks unusually as compared to previous reconstructions of magnetic activity of the Sun. However, similar temporal patterns although of greater amplitude appeared also in (Bard et al. 2000 and references therein). 


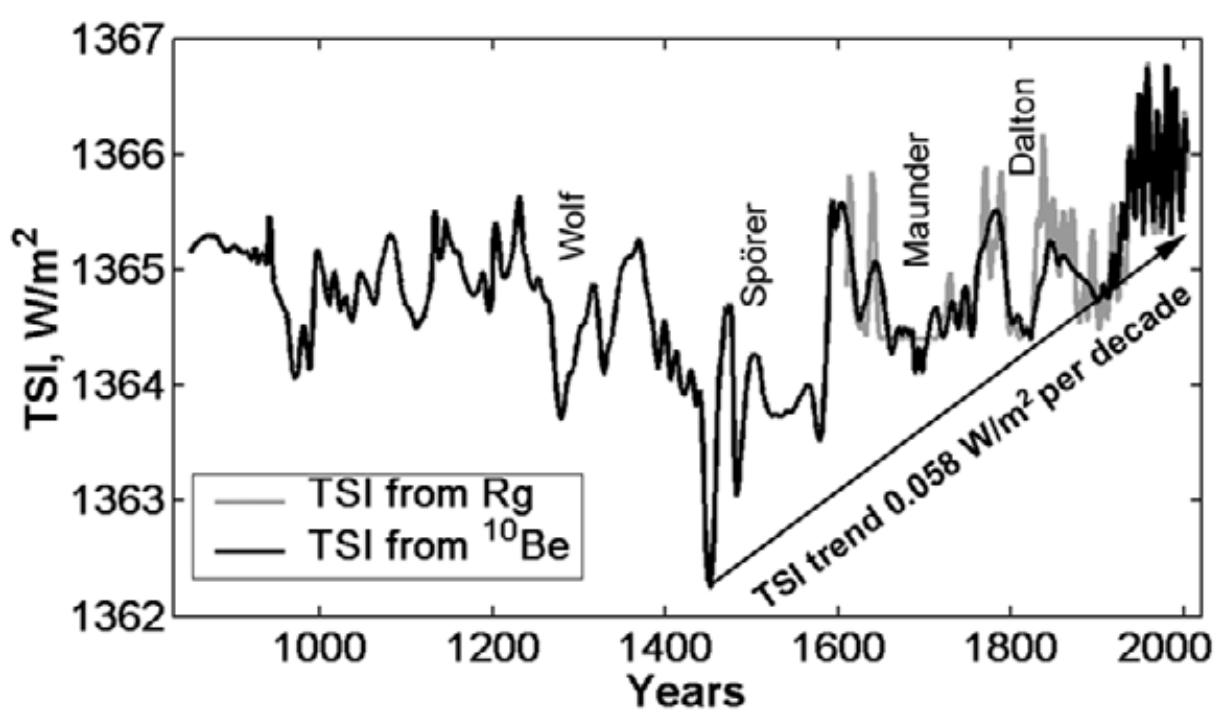

Figure 1. The TSI reconstructions using the group sunspot numbers Rg and ${ }^{10} \mathrm{Be}$ records.

There are two types of long-term changes in luminosity of the Sun appearing in the upper and lower envelopes of the cyclic TSI changes. The amplitude modulation of the cyclic TSI variations is caused by long-term changes in magnetic activity of the Sun. Slow-varying background radiation of the Sun appears in the lower envelope of cyclic TSI changes. According to the ANN reconstruction the background solar luminosity has increased about of $3.2 \mathrm{~W} / \mathrm{m}^{2}$ since 1450 . The persistent TSI change between successive minima is about of $0.058 \mathrm{~W} / \mathrm{m}^{2}$ per decade. However, between activity cycles $6,7,8$ and $16,17,18$ the upward trend amounts to $0.04 \%$ per decade. The global thermodynamic variability of the Sun on a millennium timescale resulted from changes in the efficiency of the heat transport through the convection zone and long-term changes in the solar dynamo.

\section{Acknowledgements}

This research is supported by the Federal program "Astronomy" 1105, RFBR 02-0216044 and INTAS 2001-0550.

\section{References}

Bard, E., Raisbeck, G.M., Yiou, F. \& Jouzel, J. 1997 J.Earth Planetary Sci. Lett. 150, 453.

Bard, E., Raisbeck, G.M., Yiou, F. \& Jouzel, J. 2000 Tellus 52B, 985.

Benevolenskaya, E.E., Kosovichev, A.G., \& Scherrer, P.H. 1999 Solar Phys. 190, 145.

Fröhlich, C. \& Lean, J. 1998 Geophys. Res. Lett. 25, 4377.

Hoyt, D.V. \& Schatten, K. 1998 Solar Phys. 179, 189

Lean, J., Beer, J. \& Bradley, R. 1995 Geophys. Res. Lett. 22, 3195.

Mordvinov, A.V. \& Willson, R.C. 2003 Solar Phys. 215, 5.

Solanki, S., Fligge, M. 1999 Geophys. Res. Lett. 26, 2465.

Usoskin, I.G., Mursula, K., Solanki, S., Schüssler, M. \& Alanko, K. 2004 Astron. Astrophys. 413, 745 .

Willson, R.C. \& Mordvinov, A.V. 2003 Geophys. Res. Lett. 30, 1199. 